\title{
CONJUGAL TRANSFER OF IMIPENEM RESISTANCE IN
}

\author{
Bacteroides fragilis
}

\author{
Kaori Bandoh, Kunitomo Watanabe, Yoshinori Muto, \\ YasunORI TANAKa, NaOki Kato and KazUe Ueno \\ Institute of Anaerobic Bacteriology, Gifu University School of Medicine, \\ Gifu, Japan
}

(Received for publication September 30, 1991)

\begin{abstract}
Transfer of imipenem resistance in Bacteroides fragilis was studied. Clinical isolate $B$. fragilis 10-73 was highly resistant to imipenem. Imipenem resistance was transferred from 10-73 to $B$. fragilis strain TM 4000 at a frequency of $10^{-6} /$ input recipient by a filter mating technique. The resistance could also be retransferred. $B$. fragilis 10-73 and both primary and secondary transcipients produced an imipenem-hydrolyzing metallo- $\beta$-lactamase. Acquisition of imipenem resistance correlated with the appearance of plasmid DNA with a size $(c a .13 .6 \mathrm{~kb})$ similar to that of the donor strain. TM4000 transformed by electroporation with purified DNA of the 13.6-kb plasmid pBFUK1 produced the metallo- $\beta$-lactamase and was resistant to imipenem. Transfer was resistant to DNase treatment and no transfer was seen with a sterile filtrate of the donor culture. It is suggested that gene transfer in B. fragilis has the properties of a conjugation system rather than those of transformation or transduction.
\end{abstract}

Bacteroides fragilis is an important pathogen in anaerobic infections. Clinically isolated $B$. fragilis generally produce $\beta$-lactamase and are moderately or highly resistant to penicillins and cephalosporins. Imipenem has excellent antimicrobial activity against these organisms. Resistance to imipenem is rare in B. fragilis but a few strains have been reported to be resistant ${ }^{1 \sim 3}$. We previously reported two $B$. fragilis clinical isolates, GAI-30079 and 30144 that are highly resistant to imipenem and to most other $\beta$-lactam antibiotics ${ }^{3)}$. Both strains had an imipenem-hydrolyzing $\beta$-lactamase. The enzyme activity was inhibited by EDTA. It was suggested that this $\beta$-lactamase was a metalloenzyme. Recently we found imipenemresistant $B$. fragilis 10-73 in which resistance was transferrable by a conjugation system. This study investigates the transferrability of imipenem resistance and plasmid in matings involving $B$. fragilis 10-73.

\section{Materials and Methods}

\section{Bacterial Strains}

The strains of $B$. fragilis used in this work are described in Table 1. B. fragilis 10-73, isolated from materials of a wounded infection, was resistant to imipenem, tetracycline, penicillins, cephalosporins, cephamycins, and latamoxef (Table 2). Rifampicin-resistant mutant strains, B. fragilis TM4000 and JC101 were used as recipients in the matings.

Media Used

For the liquid culture, brain heart-infusion broth (BHIB) (Becton Dickinson \& Co., Maryland, U.S.A.) supplemented with hemin $(5 \mu \mathrm{g} / \mathrm{ml})$ and yeast extract $(5 \mu \mathrm{g} / \mathrm{ml})$, and GAM broth (Nissui Seiyaku Co., Ltd., Tokyo, Japan) supplemented with hemin $(5 \mu \mathrm{g} / \mathrm{ml})$ and menadione $(0.1 \mu \mathrm{g} / \mathrm{ml})$ were used. Anaerobic minimum media were prepared using a slightly modified version of the method of VAREL and BRYANT ${ }^{4)}$.

Susceptibility Tests

The MICs were determined by an agar dilution method using modified GAM agar (Nissui Seiyaku 
Table 1. Bacteroides fragilis strains and relevant characteristics.

\begin{tabular}{cll}
\hline Strain & \multicolumn{1}{c}{ Phenotype $^{\mathrm{a}, \mathrm{b}}$} & \multicolumn{1}{c}{ Comment } \\
\hline B. fragilis $10-73$ & $\mathrm{Carb}^{\mathrm{r}}, \mathrm{Rif}^{\mathrm{s}}, \mathrm{Tet}^{\mathrm{r}}, \mathrm{His}^{-} \mathrm{Arg}^{-}$ & Clinical isolate \\
TM4000 & $\mathrm{Carb}^{\mathrm{s}}, \mathrm{Rif}^{\mathrm{r}}$ & Plasmid free \\
JC101 & $\mathrm{Carb}^{\mathrm{s}}, \mathrm{Rif}^{\mathrm{r}}, \mathrm{His}^{-} \mathrm{Arg}^{-}$ & Amino acid auxotroph of TM4000, plasmid free \\
Tc 1 & $\mathrm{Carb}^{\mathrm{r}}, \mathrm{Rif}^{\mathrm{r}}, \mathrm{His}^{-} \mathrm{Arg}^{-}, \mathrm{Tet}^{\mathrm{s}}$ & Transcipient of 10-73 $\times$ JC101 matings \\
Tc 12, Tc 30 & $\mathrm{Carb}^{\mathrm{r}}, \mathrm{Rif}^{\mathrm{r}}, \mathrm{Tet}^{\mathrm{s}}$ & Transcipient of 10-73 $\times$ TM4000 matings \\
Tf3 & Carb $^{\mathrm{r}}, \mathrm{Rif}^{\mathrm{r}}$ & TM4000 transformant containing pBFUK1 from Tc 30 \\
\hline
\end{tabular}

a Carb; carbapenem, Rif; rifampicin, Tet; tetracycline.

b $\mathrm{His}^{-} \mathrm{Arg}^{-}$; auxotrophs requiring histidine and arginige for growth.

Table 2. Susceptibility of Bacteroides fragilis $10-73$ and reference strains to various $\beta$-lactams.

\begin{tabular}{|c|c|c|c|c|c|c|c|}
\hline \multirow[b]{2}{*}{ Antibiotic } & \multicolumn{3}{|c|}{$\operatorname{MIC}(\mu \mathrm{g} / \mathrm{ml})$} & \multirow[b]{2}{*}{ Antibiotic } & \multicolumn{3}{|c|}{$\mathrm{MIC}(\mu \mathrm{g} / \mathrm{ml})$} \\
\hline & $10-73$ & $\begin{array}{c}\text { GAI- } \\
30144\end{array}$ & $\begin{array}{c}\text { ATCC } \\
25285\end{array}$ & & $10-73$ & $\begin{array}{c}\text { GAI- } \\
30144\end{array}$ & $\begin{array}{r}\text { ATCC } \\
25285\end{array}$ \\
\hline Ampicillin & 100 & 200 & 12.5 & Cefotetan & 100 & 100 & 3.13 \\
\hline Piperacillin & 50 & 50 & 3.13 & Cefbuperazone & 200 & 200 & 1.56 \\
\hline Cefazolin & 200 & $>200$ & 25 & Clavulanic acid/ & 200 & 50 & 0.78 \\
\hline Cefoperazone & $>200$ & $>200$ & 12.5 & ampicillin & & & \\
\hline Ceftizoxime & $>200$ & 100 & 3.13 & Sulbactam/cefoperazone & $>200$ & 400 & 3.13 \\
\hline Cefoxitin & 100 & 25 & 6.25 & Imipenem & 200 & 100 & 0.2 \\
\hline Cefmetazole & 100 & 100 & 6.25 & Clindamycin & 6.25 & 0.2 & 0.78 \\
\hline Latamoxef & 200 & 100 & 0.78 & Tetracycline & 100 & 12.5 & ND \\
\hline
\end{tabular}

ND: Not determined.

Co., Ltd., Tokyo, Japan). A final inoculum of $10^{6} \mathrm{cfu} / \mathrm{ml}$ was applied with a replicating device. Anaerobic cultures were incubated for 48 hours at $37^{\circ} \mathrm{C}$ in an anaerobic chamber (Model 1204; Forma Scientific, Ohio, U.S.A.).

\section{Transfer Experiment}

Matings were done by the filter mating procedures). The parental strains were grown separately in BHIB until the mid-logarithmic phase. Donor and recipient strains were mixed in $1: 10$ ratio, respectively and approximately $5 \mathrm{ml}$ of the mixture was filtered onto the surface of a $0.45 \mu \mathrm{m}$ Nalgene filter (Nalgene Co., New York, U.S.A.). For dummy mating, donor culture filtrate passed through a $0.45 \mu \mathrm{m}$ filter was used as the donor. The filter was placed on a modified GAM agar and incubated overnight at $37^{\circ} \mathrm{C}$ in the anaerobic chamber. The cells were harvested from the filter by suspension in BHIB. Aliquots were placed on selective media. Rifampicin, tetracycline and meropenem were used for selection.

\section{$\beta$-Lactamase Assay}

Cells from an overnight culture in GAM broth were suspended in $50 \mathrm{~mm}$ MOPS - KOH (pH 7.2) buffer and disrupted by sonication. Cell extracts centrifuged at $10,000 \times g$ for 15 minutes at $4^{\circ} \mathrm{C}$ were used as a crude enzyme preparation. The enzyme activity was assayed using a spectrophotometric technique ${ }^{6)}$. The reaction mixture contained a $100 \mu \mathrm{M} \beta$-lactam compound in a $200 \mathrm{mM}$ MOPS - KOH (pH 7.2) buffer and was maintained at $30^{\circ} \mathrm{C}$. Reaction was initiated by adding an aliquot of crude enzyme. Drug inactivation was monitored at the following wavelengths; benzylpenicillin $233 \mathrm{~nm}$, ampicillin $235 \mathrm{~nm}$, cephaloridine $260 \mathrm{~nm}$, cefoxitin $270 \mathrm{~nm}$, cefotaxime $264 \mathrm{~nm}$, ceftizoxime $257 \mathrm{~nm}$, cefoperazone $273 \mathrm{~nm}$, latamoxef $275 \mathrm{~nm}$, imipenem $299 \mathrm{~nm}$, and aztreonam $318 \mathrm{~nm}$.

DNA Preparation and Analysis

Routine screening for plasmid content was performed using the alkaline lysis technique ${ }^{7)}$. Purified plasmid DNA was prepared using cesium chloride-ethidium bromide ultracentrifugation ${ }^{8}$. Restriction 
endonuclease digestions were performed at $37^{\circ} \mathrm{C}$ for 2 hours in the buffers recommended by the manufacturer (Takara Shuzo Co., Ltd., Kyoto, Japan). DNA preparations were analyzed in $0.8 \%$ agarose gel by the method described by TALLY et al. ${ }^{5)}$.

\section{Transformation Experiment}

For the transformation experiment, purified plasmid DNA from B. fragilis transcipient Tc 30 was used. Transformation of $B$. fragilis TM 4000 was performed using the electrotransformation technique (Gene pulsar $^{\mathrm{TM}}$ apparatus Bio-Rad Laboratories, Richmond, CA, U.S.A.) as described by SEBALD et al. ${ }^{9)}$.

\section{Results}

The susceptibility of $B$. fragilis 10-73 is shown in Table 2. Compared to reference strain ATCC 25285, strain 10-73 was highly resistant to cephamycins, ceftizoxime, latamoxef, and imipenem. It was also resistant to ampicillin/clavulanic acid and cefoperazone/sulbactam. Its susceptibility pattern is quite similar to that of highly imipenem-resistant strain GAI-30144 which produces metallo- $\beta$-lactamase ${ }^{3)}$. Besides $\beta$-lactam antibiotics, $10-73$ was also resistant to $50 \mu \mathrm{g} / \mathrm{ml}$ of tetracycline.

To investigate the ability of 10-73 to transfer antibiotic resistance, we mated this strain with $B$. fragilis TM4000. As shown in Table 3, imipenem resistance was transferred at a frequency of $10^{-6} / \mathrm{input}$ recipient. However, transfer of tetracycline resistance was not observed (Table 3). The transcipients (Tc) obtained by primary mating were resistant to $\beta$-lactams at levels comparable to those of the donor strain 10-73, but were sensitive to tetracycline (data not shown). When plasmid DNA from twenty strains of transcipient was analyzed by electrophoresis in $0.8 \%$ agarose, it was found that the migration patterns of plasmid DNA fell into three classes (Fig. 1: Lanes 1 and 7; Type 1, Lanes 2 5; Type 2, Lane 6; Type 3) but all transcipients contained plasmid DNA which was homologous with the plasmid DNA of a similar size in the donor strain (Fig. 1; indicated by arrows).

Secondary mating was done between Tc 1, a transcipient with JC101 background and TM4000. This was because we didn't have a suitable selective marker in secondary mating between a transcipient with TM4000 background and JC101. Imipenem resistance was transferred at a frequency of $10^{-8}$ /input recipient in a primary mating between 10-73 and $\mathrm{JC101}$, and retransferred at a frequency of $10^{-5} /$ input recipient (Table 3). As with the primary mating with TM4000, common plasmid DNA was also observed in 10-73, Tc 1 and transcipients of secondary mating (data not shown). Thus, acquisition of imipenem resistance was accompanied by the appearance of plasmid DNA and was. separated from a tetracycline-resistance transfer element.

Table 3. Transfer of resistance between Bacteroides fragilis by filter mating.

\begin{tabular}{|c|c|c|c|c|}
\hline \multirow{2}{*}{ Donor } & \multirow{2}{*}{ Recipient } & \multirow{2}{*}{ Selective medium ${ }^{\mathbf{a}}$} & \multicolumn{2}{|c|}{ Transcipient } \\
\hline & & & $\begin{array}{c}\text { Frequency } \\
\text { (per/recipient) }\end{array}$ & Phenotype \\
\hline $10-73$ & TM4000 & Rif, Mepm & $10^{-6}$ & Mepm $^{r}$, Rif $^{r}$ \\
\hline $10-73$ & TM4000 & Rif, Tet & $<10^{-10}$ & $\operatorname{Tet}^{s}$ \\
\hline $10-73$ & JC101 & Rif, Mepm & $10^{-8}$ & Mepm $^{\mathrm{r}}, \mathrm{Rif}^{\mathrm{r}}, \mathrm{Tet}^{\mathrm{s}}, \mathrm{His}^{-} \mathrm{Arg}^{-}$ \\
\hline $\mathrm{Tc} 1$ & TM4000 & Rif, Mepm/AMM ${ }^{\mathrm{b}}$ & $10^{-5}$ & Mepm $^{\mathrm{r}}$, Rif ${ }^{\mathrm{r}}, \mathrm{Tet}^{\mathrm{s}}, \mathrm{His}^{+} \mathrm{Arg}^{+}$ \\
\hline
\end{tabular}

a The selective media contained rifampicin (Rif, $25 \mu \mathrm{g} / \mathrm{ml}$ ) plus appropriate antibiotics at the following concentrations $(\mu \mathrm{g} / \mathrm{ml})$ : Meropenem (Mepm, 50); tetracycline (Tet, 5).

b Colonies, which grew on selective media containing rifampicin and meropenem, were replica plated on anaerobic minimum media (AMM) and confirmed their ability to grow on AMM plate. 
Cell extracts from 10-73 had $\beta$-lactamase activity as shown in Table 4 . The enzyme hydrolyzed penicillins, oxyiminocephalosporins, cefoxitin, latamoxef, and carbapenems, but not aztreonam. Comparison of susceptibility and substrate profile suggests that resistance to a broad range of $\beta$-lactams, including carbapenems, was due to in $\beta$-lactamase activity. Cell extracts from some transcipients were also subjected to $\beta$-lactamase assay. As shown in Table $4, \beta$-lactamases from transcipients (Tc) showed a very similar substrate hydrolysis pattern as that of 10-73, but different from that of typical cephalosporinase from TM4000. It is reasonable to think that imipenem-hydrolyzing $\beta$-lactamases of transcipients are derived from the donor strain, 10-73. Substrate profiles of the enzyme from 10-73 and transcipients were almost identical to those of metallo- $\beta$-lactamase from GAI30144 (Table 4). In addition, as with GAI-30144 enzyme, their activity was strongly inhibited by EDTA $(100 \mu \mathrm{M})$, but not by clavulanic acid $(500 \mu \mathrm{M})$. There is a possibility that the enzymes from 10-73 and transcipients are identical to those from GAI30144. However, in GAI-30144 and another metallo- $\beta$-lactamase-producing $B$. fragilis, GAI30079 , imipenem resistance was never transferred to B. fragilis JC101 (data not shown).

Plasmid DNA was purified using cesium chloride-ethidium bromide ultracentrifugation from $\mathrm{Tc} 30$, one of the transcipients which contained a minimal number of DNA bands in electrophoresis (example Fig. 1: Lanes 1 and 7). Analysis of the products of restriction enzyme cleavage of plasmid DNA confirmed the presence of a single plasmid of

Fig. 1. Agarose gel analysis of DNA in parents and transcipients of Bacteroides fragilis.

Lanes 1 7: transcipients from 10-73 $\times$ TM4000 matings, 8: TM4000 (recipient), 9: 10-73 (donor), 10 Hind III digests of lambda-DNA

Plasmid bands which were observed in all transcipients and the donor strain, 10-73, are indicated by arrows at the left.

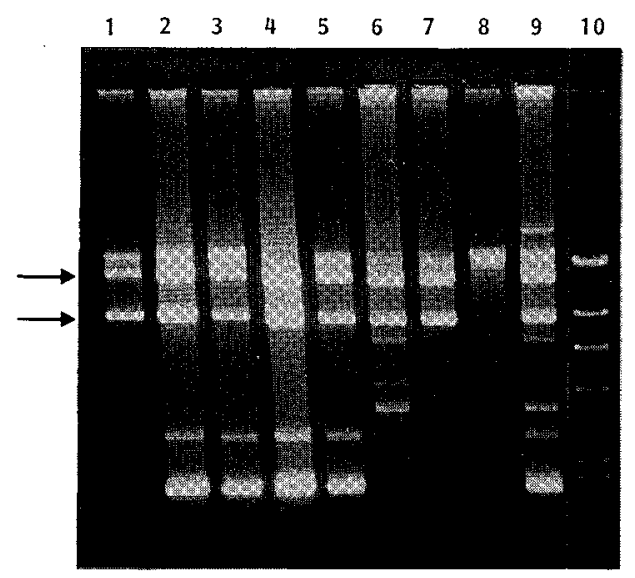

Table 4. $\beta$-Lactamase activities of cell extracts from Bacteroides fragilis 10-73, transcipients, transformant and a reference strain.

\begin{tabular}{lccccc}
\hline \multirow{2}{*}{ Substrate } & \multicolumn{5}{c}{ Rate of hydrolysis ${ }^{\mathrm{a}}(\%)$} \\
\cline { 2 - 6 } & $10-73$ & $\mathrm{Tc}^{\mathrm{b}} 12$ & Tc 30 & $\mathrm{Tf}^{\mathrm{c}} 3$ & 30144 \\
\hline Benzylpenicillin & 252 & 161 & 285 & 354 & 498 \\
Ampicillin & 178 & 104 & 159 & 236 & 90 \\
Cephaloridine & 100 & 100 & 100 & 100 & 100 \\
Cefoperazon & 334 & 228 & 181 & 247 & 206 \\
Cefotaxime & 90 & 84 & 62 & 89 & 94 \\
Ceftizoxime & 147 & 162 & 136 & 147 & 265 \\
Cefoxitin & 8 & 7 & 6 & 11 & 11 \\
Latamoxef & 164 & 146 & 78 & 71 & 108 \\
Imipenem & 127 & 120 & 102 & 99 & 182 \\
Meropenem & 184 & 146 & 111 & 147 & - \\
Aztreonam & ND & ND & ND & ND & ND \\
\hline
\end{tabular}

a The rate of hydrolysis was expressed using cephaloridine as $100 \%$.

b Tc: transcripient of 10-73 $\times$ TM4000.

c Tf: transformant of TM4000 with pBFUK1 from Tc 30 .

d ND: Not detectable. 
approximately 13.6 kilobase pairs $(\mathrm{kb})$, designated pBFUK1. Plasmid pBFUK1 was subjected to electrotransformation into TM4000. Imipenem-resistant transformants (Tf) were obtained at a frequency of $10^{-5}$ transformant/survivor. Eight strains selected randomly from those transformants were analyzed for their plasmid content and it was found that all strains contained a plasmid band which seemed to be the plasmid pBFUK1. Crude enzyme prepared from a transformant (Tf) had a similar substrate hydrolysis pattern as that of Tc 30 (Table 4). The enzyme was also very sensitive to EDTA, but not to clavulanic acid. These results suggest that plasmid pBFUK1, which was present in Tc 30 and consequently in 10-73, bore imipenem resistance gene coding for metallo- $\beta$-lactamase.

To investigate the nature of transfer, we performed mating on a GAM plate containing $1 \mathrm{mg} / \mathrm{ml}$ of DNase. Dummy mating, using donor (10-73), was performed using a culture sterile filtrate. Transfer of imipenem resistance was not prevented by the presence of DNase and no transfer was observed in mating between a sterile filtrate of the donor culture and TM4000. Therefore, gene transfer by B. fragilis 10-73 was suggested to have the properties of a conjugation system rather than those of transformation or transduction.

\section{Discussion}

Imipenem has excellent antimicrobial activity against a broad range of bacterial species. An increasing number of research groups has recently been involved with impenem resistance. Species studied include Xanthomonas maltophilia ${ }^{10)}$, Flavobacterium odoratum ${ }^{11)}$, Legionella gormanii ${ }^{12)}$, Serratia marcescens ${ }^{13)}$, mutant strains of Aeromonas hydrophilia ${ }^{14)}$, and B. fragili ${ }^{1}{ }^{13}$. Resistance to imipenem is associated with imipenem-hydrolyzing metallo- $\beta$-lactamase in those species. In Pseudomonas aeruginosa, imipenem resistance is known to be associated with a specific decrease in imipenem permeability ${ }^{15)}$, but recently it has also been shown to be associated with an imipenem-hydrolyzing metallo- $\beta$-lactamase ${ }^{16)}$. Imipenemhydrolyzing metallo- $\beta$-lactamases thus play an important role in many imipenem-resistant organisms. In this study, we demonstrated the intraspecific transfer of imipenem-resistance within $B$. fragilis from a highly imipenem-resistant $B$. fragilis $10-73$, resistance being due to the production of an imipenemhydrolyzing metallo- $\beta$-lactamase. Electrotransformation studies indicated that the transfer of imipenem resistance was associated with that of the conjugative plasmid pBFUK1. Others, such as, WATANABE et $a l$., have recently demonstrated that the metallo- $\beta$-lactamase from $P$. aeruginosa is plasmid-mediated and that the plasmid is transferrable ${ }^{16}$. The enzyme encoded by pMS350 from $P$. aeruginosa has a similar substrate and inhibitor profile as that of the $B$. fragilis 10-73 enzyme. It would be of great interest to define the relationship between the $\beta$-lactamase gene on pMS350 and pBFUK1, as well as their products.

In $B$. fragilis, imipenem-hydrolyzing $\beta$-lactamases have been reported by YoTsuI et al. $(\mathrm{G} 237)^{1)}$, Cuchural et al. (TAL2480) ${ }^{2)}$, and BANDOH et al. (GAI-30144) ${ }^{3)}$. On the basis of the properties of their substrate and inhibitor profiles, these three enzymes and the 10-73 enzyme can be classified as type II oxyiminocephalosporinase (CXase) by MrTsuHASHI et al. ${ }^{11)}$, as they are quite similar to one another. It is tempting to speculate that these imipenem-hydrolyzing enzymes found in $B$. fragilis were derived from the same ancestor. In GAI-30144, however, the transfer of imipenem resistance was not observed as described in this paper. A gene coding for the metallo- $\beta$-lactamase from TAL2480 has been shown to be present in the chromosome ${ }^{17)}$, this is in contrast to the plasmid of our strain 10-73. These observations suggest a variety of genes coding for imipenem-hydrolyzing $\beta$-lactamase in $B$. fragilis.

The precise mechanism of the resistance transfer process in $B$. fragilis 10-73 remains unknown, but our data suggest that imipenem resistance transfer is independent of the tetracycline-resistance transfer element. Plasmid pBFUK1 seems to be self-transferrable. However, the size of the plasmid is rather small compared to most of the self-transferrable plasmids and there is a possibility that the plasmid is mobilized.

In Japan, imipenem resistance in B. fragilis is currently rare. According to research at our institute, 6 of 433 (about $1.4 \%$ ) strains of $B$. fragilis, which were isolated from clinical specimens over the 1986 to 1989 period, were resistant to $8 \mu \mathrm{g} / \mathrm{ml}$ of imipenem. However, the present observation that the transfer of 
a plasmid-bearing metallo- $\beta$-lactamase can naturally occur is of great significance. This is because transferrable plasmids play an important role in the rapid dissemination of resistance to antibiotics.

\section{References}

1) Yotsui, A.; S. Minami, M. Inoue \& S. Mitsuhashi: Properties of novel $\beta$-lactamase produced by Bacteroides fragilis. Antimicrob. Agents Chemother. 24: 925 929, 1983

2) Cuchural, G. J., Jr.; M. H. Malamy \& F. P. Tally: $\beta$-Lactamase-mediated imipenem resistance in Bacteroides fragilis. Antimicrob. Agents Chemother. 30: 645 648, 1986

3) Bandoh, K.; Y. Muto, K. Watanabe, N. Katoh \& K. Ueno: Biochemical properties and purification of metallo- $\beta$-lactamase from Bacteroides fragilis. Antimicrob. Agents Chemother. 35: 371 372, 1991

4) VAReL, V. H. \& M. P. BRyant: Nutritional features of Bacteroides fragilis subsp. fragilis. Appl. Microbiol. 28: $251 \sim 257,1974$

5) Tally, F. P.; D. R. Snydman, S. L. Gorbach \& M. H. Malamy: Plasmid-mediated, transferable resistance to clindamycin and erythromycin in Bacteroides fragilis. J. Infect. Dis. 139: 83 88, 1979

6) Neu, H. C.: Antibiotic inactivating enzymes and bacterial resistance. In Antibiotics in Laboratory Medicine. 2nd Ed. Ed., V. LORian, pp. 757 789, The Williams \& Wilkins Co., 1986

7) Manitatis, T.; E. F. Fritsch \& J. Sambrook: Molecular Cloning-A Laboratory Manual. Cold Spring Harbor Laboratory, 1982

8) Clewell, D. B. \& D. R. Helinski: Properties of a supercoiled deoxyribonucleic acid - protein relaxation complex and strand specificity of the relaxation event. Biochemistry 9: 4428 4440, 1970

9) Sebald, M.; G. Reysset \& J. Breuil: What's new in 5-nitroimidasole resistance in the Bacteroides fragilis group? In Clinical and Molecular Aspects of Anaerobes. Ed., S. P. Borriello, pp. $217 \sim 225$, Wrightson Biomedical Publishing Ltd., 1990

10) Saino, Y.; F. Kobayashi, M. Inoue \& S. Mitsuhashi: Purification and properties of inducible penicillin $\beta$-lactamase isolated from Pseudomonas maltophilia. Antimicrob. Agents Chemother. 22: 564 570, 1982

11) Sato, K; T. Fujn, R. Okamoto, M. Inoue \& S. Mitsuhashi: Biochemical properties of $\beta$-lactamase produced by Flavobacterium odoratum. Antimicrob. Agents Chemother. 27: 612 614, 1985

12) Fuji, T.; K. Sato, K. Miyata, M. Inoue \& S. Mitsuhashi: Biochemical properties of $\beta$-lactamase produced by Legionella gormanii. Antimicrob. Agents Chemother. 29: $925 \sim 926,1986$

13) YANG, Y.; P. WU \& D. M. LIVERMORE: Biochemical characterization of a $\beta$-lactamase that hydrolyzes penems and carbapenems from two Serratia marcescens isolates. Antimicrob. Agents Chemother. 34: 755 758, 1990

14) IAConis, J. P. \& C. C. SANders: Purification and characterization of inducible $\beta$-lactamases in Aeromonas spp. Antimicrob. Agents Chemother. 34: 44 51, 1990

15) Büscher, K.-H.; W. Cullmann, W. Dick \& W. Opferkuch: Imipenem resistance in Pseudomonas aeruginosa resulting from diminished expression of an outer membrane protein. Antimicrob. Agents Chemother. 31: 703 708, 1987

16) Watanabe, M.; S. Iyobe, M. Inoue \& S. Mitsuhashi: Transferable imipenem resistance in Pseudomonas aeruginosa. Antimicrob. Agents Chemother, 35: 147 151, 1991

17) Thompson, J. S. \& M. H. Malamy: Sequencing the gene for an imipenem-cefoxitin-hydrolyzing enzyme (CfiA) from Bacteroides fragilis TAL2480 reveals strong similarity between CfiA and Bacillus cereus $\beta$-lactamase II. J. Bacteriol. 172: $2584 \sim 2593,1990$ 